\title{
Top degree coefficients of the Denumerant
}

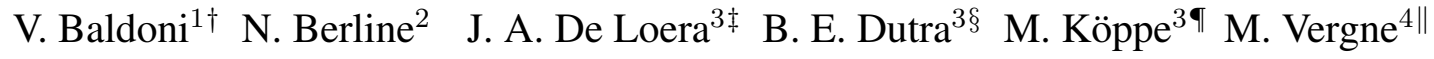 \\ ${ }^{1}$ Dipartimento di Matematica, Università degli studi di Roma "Tor Vergata", Roma, Italy \\ ${ }^{2}$ Centre de Mathématiques Laurent Schwartz, École Polytechnique, Palaiseau, France \\ ${ }^{3}$ Department of Mathematics, University of California, Davis, CA, USA \\ ${ }^{4}$ Institut de Mathématiques de Jussieu, Théorie des Groupes, Paris, France
}

\begin{abstract}
For a given sequence $\boldsymbol{\alpha}=\left[\alpha_{1}, \alpha_{2}, \ldots, \alpha_{N}, \alpha_{N+1}\right]$ of $N+1$ positive integers, we consider the combinatorial function $E(\boldsymbol{\alpha})(t)$ that counts the nonnegative integer solutions of the equation $\alpha_{1} x_{1}+\alpha_{2} x_{2}+\cdots+\alpha_{N} x_{N}+$ $\alpha_{N+1} x_{N+1}=t$, where the right-hand side $t$ is a varying nonnegative integer. It is well-known that $E(\boldsymbol{\alpha})(t)$ is a quasipolynomial function of $t$ of degree $N$. In combinatorial number theory this function is known as the denumerant. Our main result is a new algorithm that, for every fixed number $k$, computes in polynomial time the highest $k+1$ coefficients of the quasi-polynomial $E(\boldsymbol{\alpha})(t)$ as step polynomials of $t$. Our algorithm is a consequence of a nice poset structure on the poles of the associated rational generating function for $E(\boldsymbol{\alpha})(t)$ and the geometric reinterpretation of some rational generating functions in terms of lattice points in polyhedral cones. Experiments using a MAPLE implementation will be posted separately.

Résumé. Considérons une liste $\boldsymbol{\alpha}=\left[\alpha_{1}, \alpha_{2}, \ldots, \alpha_{N+1}\right]$ de $N+1$ entiers positifs. Le dénumérant $E(\boldsymbol{\alpha})(t)$ est la fonction qui compte le nombre de solutions en entiers positifs ou nuls de l'équation $\sum_{i=1}^{N+1} x_{i} \alpha_{i}=t$, où $t$ varie dans les entiers positifs ou nuls. Il est bien connu que cette fonction est une fonction quasi-polynomiale de $t$, de degré $N$. Nous donnons un nouvel algorithme qui calcule, pour chaque entier fixé $k$ (mais $N$ n'est pas fixé), les $k+1$ plus hauts coefficients du quasi-polynôme $E(\boldsymbol{\alpha})(t)$ en termes de fonctions en dents de scie. Notre algorithme utilise la structure d'ensemble partiellement ordonné des pôles de la fonction génératrice de $E(\boldsymbol{\alpha})(t)$. Les $k+1$ plus hauts coefficients se calculent à l'aide de fonctions génératrices de points entiers dans des cônes polyèdraux de dimension inférieure ou égale à $k$.
\end{abstract}

Keywords: Denumerants, Ehrhart quasi-polynomials, partitions, polynomial-time algorithm

\section{Introduction}

Let $\boldsymbol{\alpha}=\left[\alpha_{1}, \alpha_{2}, \ldots, \alpha_{N}, \alpha_{N+1}\right]$ be a sequence of positive integers. If $t$ is a non-negative integer, we denote by $E(\boldsymbol{\alpha})(t)$ the number of solutions in nonnegative integers of the equation $\sum_{i=1}^{N+1} \alpha_{i} x_{i}=$

\footnotetext{
${ }^{\dagger}$ Partially supported by PRIN project, MIUR

$\ddagger$ Partially supported by NSF grant DMS-0914107

§Partially supported by NSF-VIGRE grant DMS-0636297

T Partially supported by NSF grant DMS-0914873

" All authors are grateful for support received through a SQuaRE program at the American Institute of Mathematics
}

1365-8050 @ 2013 Discrete Mathematics and Theoretical Computer Science (DMTCS), Nancy, France 
$t$. In other words, $E(\boldsymbol{\alpha})(t)$ is the same as the number of partitions of the number $t$ using the parts $\alpha_{1}, \alpha_{2}, \ldots, \alpha_{N}, \alpha_{N+1}$ (with repetitions allowed). This paper presents a new algorithm to compute individual coefficients of this quasipolynomial function and uncovers new structure that allows to describe their periodic nature. Let us begin with some background and history before stating the precise results:

The combinatorial function $E(\boldsymbol{\alpha})(t)$ was called by J. Sylvester the denumerant. The denumerant $E(\boldsymbol{\alpha})(t)$ has a beautiful structure and it has been known since the times of Cayley and Sylvester that $E(\boldsymbol{\alpha})(t)$ is in fact a quasi-polynomial, i.e., it can be written in the form $E(\boldsymbol{\alpha})(t)=\sum_{i=0}^{N} E_{i}(t) t^{i}$, where $E_{i}(t)$ is a periodic function of $t$ (a more precise description of the periods of the coefficients $E_{i}(t)$ will be given later). In other words, there exists a positive integer $Q$ such that for $t$ in the coset $q+Q \mathbb{Z}$, the function $E(\boldsymbol{\alpha})(t)$ coincides with a polynomial function of $t$. The study of the coefficients $E_{i}(t)$, in particular determining their periodicity, is a problem that has occupied various authors and it is the key focus of our investigations here.

Sylvester and Cayley first showed that the function can be written in the form $A(t)+U(t)$, where $A(t)$ is a polynomial in $t$ of degree $N$ and $U(t)$ is a periodic function of period the least common multiple of $a_{1}, \ldots, a_{r}$ (see [5, 6] and references therein). In 1943, E.T. Bell gave a simpler proof and remarked that the period $Q$ is in the worst case given by the least common multiple of the $a_{i}$, but in general it can be smaller. A classical observation that goes back to I. Schur is that when the list $\boldsymbol{\alpha}$ consist of relatively prime numbers, then asymptotically $E(\boldsymbol{\alpha})(t) \approx \frac{t^{N}}{N ! \alpha_{1} \alpha_{2} \cdots \alpha_{N+1}}$ as the number $t \rightarrow \infty$.

Thus, in particular, there is a large enough integer $F$ such that for any $t \geq F, E(\boldsymbol{\alpha})(t)>0$ and there is a largest $t$ for which $E(\boldsymbol{\alpha})(t)=0$. Let us give a simple example:

Example 1.1. Let $\boldsymbol{\alpha}=[6,2,3]$. Then on each of the cosets $q+6 \mathbb{Z}$, the function $E(\boldsymbol{\alpha})(t)$ coincides with a polynomial $E^{[q]}(t)$. Here are the corresponding polynomials.

$$
\begin{array}{lll}
E^{[0]}(t)=\frac{1}{72} t^{2}+\frac{1}{4} t+1, & E^{[1]}(t)=\frac{1}{72} t^{2}+\frac{1}{18} t-\frac{5}{72}, & E^{[2]}(t)=\frac{1}{72} t^{2}+\frac{7}{36} t+\frac{5}{9}, \\
E^{[3]}(t)=\frac{1}{72} t^{2}+\frac{1}{6} t+\frac{3}{8}, & E^{[4]}(t)=\frac{1}{72} t^{2}+\frac{5}{36} t+\frac{2}{9}, & E^{[5]}(t)=\frac{1}{72} t^{2}+\frac{1}{9} t+\frac{7}{72} .
\end{array}
$$

Naturally, the function $E(\boldsymbol{\alpha})(t)$ is equal to 0 if $t$ does not belong to the lattice $\sum_{i=1}^{N+1} \mathbb{Z} \alpha_{i} \subset \mathbb{Z}$ generated by the integers $\alpha_{i}$. So if $g$ is the greatest common divisor of the $\alpha_{i}$ (which can be computed in polynomial time), and $\boldsymbol{\alpha} / g=\left[\frac{\alpha_{1}}{g}, \frac{\alpha_{2}}{g}, \ldots, \frac{\alpha_{N+1}}{g}\right]$ the formula $E(\boldsymbol{\alpha})(g t)=E(\boldsymbol{\alpha} / g)(t)$ holds, and we may assume that the numbers $\alpha_{i}$ span $\mathbb{Z}$ without changing the complexity of the problem. In other words, we may assume that the greatest common divisor of the $\alpha_{i}$ is equal to 1 .

Our primary concern is how to compute $E(\boldsymbol{\alpha})(t)$. This problem has received a lot of attention. Computing the denumerant $E(\boldsymbol{\alpha})(t)$ as a close formula or evaluating it for specific $t$ is relevant in several other areas of mathematics. In the combinatorics literature the denumerant has been studied extensively (see e.g., [5, 7, 11, 13] and the references therein). In combinatorial number theory and the theory of partitions, the problem appears in relation to the Frobenius problem or the coin-change problem of finding the largest value of $t$ with $E(\boldsymbol{\alpha})(t)=0$ (see [9, 10, 12] for details and algorithms). Authors in the theory of numerical semigroups have also investigated the so called gaps of the function, which are values of $t$ for which $E(\boldsymbol{\alpha})(t)=0$, i.e., those positive integers $t$ which cannot be represented by the $\alpha_{i}$. For $N=1$ the number of gaps is $\left(\alpha_{1}-1\right)\left(\alpha_{2}-1\right) / 2$ but for larger $N$ the problem is quite difficult.

Unfortunately, computing $E(\boldsymbol{\alpha})(t)$ or evaluating it are very challenging computational problems. Even deciding whether $E(\boldsymbol{\alpha})(t)>0$ for a given $t$, is a well-known (weakly) NP-hard problem. Computing $E(\boldsymbol{\alpha})(t)$, i.e., determining the number of solutions for a given $t$, is $\# P$-hard. Computing the Frobenius 
number is also known to be NP-hard [12]. Likewise, for a given coset $q+Q \mathbb{Z}$, computing the polynomial $E^{[q]}(t)$ is NP-hard. Despite the difficulty to compute the function, in some special cases one can compute information efficiently. For example, the Frobenius number can be computed in polynomial time when $N+1$ is fixed [10]. At the same time for fixed $N+1$ one can compute $E(\boldsymbol{\alpha})(t)$ in polynomial time as a special case of a well-known result of Barvinok [2]. There are several papers exploring the practical computation of the Frobenius numbers (see e.g., [9] and the many references therein).

These wonderful results for fixed $N$ were achieved using a powerful geometric interpretation of $E(\boldsymbol{\alpha})(t)$ (which was the original way we encountered the problem): The function $E(\boldsymbol{\alpha})(t)$ can also be thought of as the number of integral points in the $N$-dimensional simplex in $\mathbb{R}^{N+1}$ defined by

$$
\Delta_{\boldsymbol{\alpha}}=\left\{\left[x_{1}, x_{2}, \ldots, x_{N}, x_{N+1}\right]: x_{i} \geq 0, \sum_{i=1}^{N+1} \alpha_{i} x_{i}=t\right\}
$$

with rational vertices $\mathbf{s}_{i}=\left[0, \ldots, 0, \frac{t}{\alpha_{i}}, 0, \ldots, 0\right]$. In this context, $E(\boldsymbol{\alpha})(t)$ is a very special case of the Ehrhart function (in honor of French mathematician Eugène Ehrhart who started its study [8]). Ehrhart functions count the lattice points inside a convex polytope $P$ as it is dilated $t$ times. All of the results we mentioned about $E(\boldsymbol{\alpha})(t)$ are in fact special cases of theorems from Ehrhart theory [4]. For example, the asymptotic result of I. Schur can be recovered from seeing that the highest-degree coefficient of $E(\boldsymbol{\alpha})(t)$ is just the normalized $N$-dimensional volume of the simplex $\Delta_{\boldsymbol{\alpha}}$. Our coefficients are just special cases of Ehrhart coefficients.

This paper is about the computation of $E(\boldsymbol{\alpha})(t)$ and in particular its coefficients. Here are our main results:

It is clear that the leading coefficient is given by Schur's result. Our main theorem recovers explicit formulas for other coefficients.

Theorem 1.2. Given any fixed integer $k$, there is a polynomial time algorithm to compute the highest $k+1$ degree terms of the quasi-polynomial $E(\boldsymbol{\alpha})(t)$, that is

$$
\operatorname{Top}_{k} E(\boldsymbol{\alpha})(t)=\sum_{i=0}^{k} E_{N-i}(t) t^{N-i}
$$

The coefficients are recovered as step polynomial functions of $t$.

Note that the number $Q$ of cosets for $E(\boldsymbol{\alpha})(t)$ can be exponential in the binary encoding size of the problem, and thus it is impossible to list, in polynomial time, the polynomials $E^{[q]}(t)$ for all the cosets $q+$ $Q \mathbb{Z}$. That is why to obtain a polynomial time algorithm, the output is presented in the format of step polynomials, which we now explain:

(i) We first define the function $\{s\}=s-\lfloor s\rfloor \in[0,1)$ for $s \in \mathbb{R}$, where $\lfloor s\rfloor$ denotes the largest integer smaller or equal to $s$. The function $\{s+1\}=\{s\}$ is a periodic function of $s$ modulo 1 .

(ii) If $r$ is rational with denominator $q$, the function $T \mapsto\{r T\}$ is a function of $T \in \mathbb{R}$ periodic modulo $q$. A function of the form $T \mapsto \sum_{i} c_{i}\left\{r_{i} T\right\}$ will be called a step linear function. If all the $r_{i}$ have a common denominator $q$, this function is periodic modulo $q$. 
(iii) Then consider the algebra generated over $\mathbb{Q}$ by such functions on $\mathbb{R}$. An element $\phi$ of this algebra can be written (not in an unique way) as

$$
\phi(T)=\sum_{l=1}^{L} c_{l} \prod_{j=1}^{J_{l}}\left\{r_{l, j} T\right\}^{n_{l}, j} .
$$

Such a function $\phi(T)$ will be called a step polynomial.

(iv) We will say that the step polynomial $\phi$ is of degree $u$ if $\sum_{j} n_{j} \leq u$ for all set of indices $I$ occurring in the formula for $\phi$. We will say that $\phi$ is of period $q$ if all the rational numbers $r_{j}$ have common denominator $q$.

It must be stress that evaluating these expressions can be done very fast. Moreover, one can also see that the step polynomial representation is much more economical than writing the individual polynomials for each coset of the period. For example instead of six polynomial "pieces" for $E(\boldsymbol{\alpha})(t)$ we can simply write a single step polynomial:

$$
\frac{1}{72} t^{2}+\left(\frac{1}{4}-\frac{\left\{-\frac{t}{3}\right\}}{6}-\frac{\left\{\frac{t}{2}\right\}}{6}\right) t+\left(1-\frac{3}{2}\left\{-\frac{t}{3}\right\}-\frac{3}{2}\left\{\frac{t}{2}\right\}+\frac{1}{2}\left(\left\{-\frac{t}{3}\right\}\right)^{2}+\left\{-\frac{t}{3}\right\}\left\{\frac{t}{2}\right\}+\frac{1}{2}\left(\left\{\frac{t}{2}\right\}\right)^{2}\right)
$$

We must remark our results come after an earlier result of Barvinok [3] who first proved a similar theorem valid for all simplices. Also in [1], the authors presented a polynomial-time algorithm of to compute the coefficient functions of $\operatorname{Top}_{k} E(P)(t)$ for any simple polytope $P$ (given by its rational vertices) in the form of step polynomials defined as above. We note that both of these earlier papers use the geometry of the problem very strongly; instead our algorithm is different as it uses more of the number-theoretic structure of the special case at hand. We must stress a marked advantage of our algorithms over the work in [3]: We compute using the step polynomials all the possibilities of $E^{[q]}(t)$ while [3] recovers a single piece for given $q$. More important, our new algorithm is much easier to implement.

The new algorithm uses directly the residue theorem in one complex variable, which can be applied more efficiently as a consequence of a rich poset structure on the set of poles of the associated rational generating function for $E(\boldsymbol{\alpha})(t)$ (see Subsection 2.2). The other important ingredient used in the efficient computation of the top coefficients is the reinterpretation of some generating functions in terms of lattice points in cones. This allows us to apply the polynomial-time signed cone decomposition of Barvinok for simplicial cones of fixed dimension $k[2]$.

\section{The Residue formula for $E(\boldsymbol{\alpha})(t)$}

Let us begin by fixing some notation. If $\omega(z) \mathrm{d} z$ is a meromorphic one form on $\mathbb{C}$, with a pole at $z=\zeta$, we write

$$
\operatorname{Res}_{z=\zeta} \omega(z) \mathrm{d} z=\frac{1}{2 i \pi} \int_{C_{\zeta}} \omega(z) \mathrm{d} z
$$

where $C_{\zeta}$ is a small circle around the pole $\zeta$. If $\phi(z)=\sum_{k \geq k_{0}} \phi_{k} z^{k}$ is a Laurent series in $z$, we denote by $\operatorname{res}_{z=0}$ the coefficient of $z^{-1}$ of $\phi(z)$. Cauchy's formula implies that $\operatorname{res}_{z=0} \phi(z)=\operatorname{Res}_{z=0} \phi(z) \mathrm{d} z$. 


\subsection{A residue formula for $E(\alpha)(t)$.}

Let $\boldsymbol{\alpha}=\left[\alpha_{1}, \alpha_{2}, \ldots, \alpha_{N+1}\right]$ be our list of integers. Define

$$
F(\boldsymbol{\alpha})(z):=\frac{1}{\prod_{i=1}^{N+1}\left(1-z^{\alpha_{i}}\right)} .
$$

Denote by $\mathcal{P}=\bigcup_{i=1}^{N+1}\left\{\zeta \in \mathbb{C}: \zeta^{\alpha_{i}}=1\right\}$ the set of poles of the meromorphic function $F(\boldsymbol{\alpha})$ and by $p(\zeta)$ the order of the pole $\zeta$ for $\zeta \in \mathcal{P}$.

Note that because the $\alpha_{i}$ have greatest common divisor 1 , we have $\zeta=1$ as a pole of order $N+1$, and the other poles have order strictly less.

Theorem 2.1. Let $\boldsymbol{\alpha}=\left[\alpha_{1}, \alpha_{2}, \ldots, \alpha_{N+1}\right]$ be a list of integers with greatest common divisor equal to 1 , and let

$$
F(\boldsymbol{\alpha})(z):=\frac{1}{\prod_{i=1}^{N+1}\left(1-z^{\alpha_{i}}\right)} .
$$

Ift is a non-negative integer, then

$$
E(\boldsymbol{\alpha})(t)=-\sum_{\zeta \in \mathcal{P}} \operatorname{Res}_{z=\zeta} z^{-t-1} F(\boldsymbol{\alpha})(z) \mathrm{d} z
$$

and the $\zeta$-term of this sum is a quasi-polynomial function of $t$ with degree less than or equal to $p(\zeta)-1$.

Proof. For $|z|<1$, we write $\frac{1}{1-z^{\alpha_{i}}}=\sum_{u=0}^{\infty} z^{u \alpha_{i}}$ so that $F(\boldsymbol{\alpha})(z)=\sum_{t \geq 0} E(\boldsymbol{\alpha})(t) z^{t}$.

For a small circle $|z|=\epsilon$ of radius $\epsilon$ around 0 , the integral of $z^{k} \mathrm{~d} z$ is equal to 0 except if $k=-1$, when it is $2 i \pi$. Thus

$$
E(\boldsymbol{\alpha})(t)=\frac{1}{2 i \pi} \int_{|z|=\epsilon} z^{-t} F(\boldsymbol{\alpha})(z) \frac{\mathrm{d} z}{z}=\frac{1}{2 i \pi} \int_{|z|=\epsilon} z^{-t} \prod_{i=1}^{N+1} \frac{1}{\left(1-z^{\alpha_{i}}\right)} \frac{d z}{z} .
$$

Because the $\alpha_{i}$ are positive integers, and $t$ a non-negative integer, there are no residues at $z=\infty$ and we obtain equation (2.1) by applying the residue theorem.

Write $E_{\zeta}(t):=-\operatorname{Res}_{z=\zeta} z^{-t} F(\boldsymbol{\alpha})(z) \frac{\mathrm{d} z}{z}$; then the dependence in $t$ of $E_{\zeta}(t)$ comes from the expansion of $z^{-t}$ near $z=\zeta$. We write $z=\zeta+y$, so that $E_{\zeta}(t)=-\operatorname{Res}_{y=0}(\zeta+y)^{-t} F(\boldsymbol{\alpha})(\zeta+y) \frac{\mathrm{d} y}{\zeta+y}$. As the pole of $F(\boldsymbol{\alpha})(\zeta+y)$ at $y=0$ is of order $p(\zeta)$, to compute the residue at $y=0$, we only need to expand in $y$ the function $(\zeta+y)^{-t-1}$ and take the coefficient of $y^{p(\zeta)-1}$. Now for $k=t+1$ the function $(\zeta+y)^{-k}=\zeta^{-k}-k \zeta^{-k-1} y+\cdots$ and we can easily check that the dependence in $t$ of our residue is quasi-polynomial with degree less than or equal to $p(\zeta)-1$. We thus obtain the result.

\subsection{The poset of the high-order poles}

Given an integer $0 \leq k \leq N$, we partition the set of poles $\mathcal{P}$ in two disjoint sets according to the order of the pole:

$$
\mathcal{P}_{>N-k}=\{\zeta: p(\zeta) \geq N+1-k\}, \quad \mathcal{P}_{\leq N-k}=\{\zeta: p(\zeta) \leq N-k\} .
$$

According to the disjoint decomposition $\mathcal{P}=\mathcal{P}_{\leq N-k} \cup \mathcal{P}_{>N-k}$, we write 


$$
E_{\mathcal{P}_{>N-k}}(t)=-\sum_{\zeta \in \mathcal{P}_{>N-k}} \operatorname{Res}_{z=\zeta} z^{-t-1} F(\boldsymbol{\alpha})(z) \mathrm{d} z
$$

and

$$
E_{\mathcal{P}_{\leq N-k}}(t)=-\sum_{\zeta \in \mathcal{P}_{\leq N-k}} \operatorname{Res}_{z=\zeta} z^{-t-1} F(\boldsymbol{\alpha})(z) \mathrm{d} z .
$$

The following proposition is a direct consequence of Theorem 2.1.

Proposition 2.2. We have

$$
E(\boldsymbol{\alpha})(t)=E_{\mathcal{P}_{>N-k}}(t)+E_{\mathcal{P}_{\leq N-k}}(t),
$$

where the function $E_{\mathcal{P}_{\leq N-k}}(t)$ is a quasi-polynomial function of t of degree in $t$ strictly less than $N-k$.

Thus for the purpose of computing $\operatorname{Top}_{k} E(\boldsymbol{\alpha})(t)$ it is sufficient to compute the function $E_{\mathcal{P}_{>N-k}}(t)$. This function is computable in polynomial time, as stated in the following theorem that implies is Theorem 1.2

Theorem 2.3. Let $k$ be a fixed number. Then the coefficient functions of the quasi-polynomial function $E_{\mathcal{P}_{>N-k}}(t)$ are computable in polynomial time as step polynomials of $t$.

We prove the theorem in the rest of this section and the next.

We first rewrite our set $\mathcal{P}_{>N-k}$. Note that if $\zeta$ is a pole of order $\geq p$, this means that there exist at least $p$ elements $\alpha_{i}$ in the list $\boldsymbol{\alpha}$ so that $\zeta^{\alpha_{i}}=1$. But if $\zeta^{\alpha_{i}}=1$ for a set $I \subseteq\{1, \ldots, N+1\}$ of indices $i$, this is equivalent to the fact that $\zeta^{f}=1$, for $f$ the greatest common divisor of the elements $\alpha_{i}, i \in I$.

Now let $\mathcal{I}_{>N-k}$ be the set of index sets that correspond to sublists of $\boldsymbol{\alpha}$ of length greater than $N-k$. Note that when $k$ is fixed, the cardinality of $\mathcal{I}_{>N-k}$ is a polynomial function of $N$. For each subset $I \in \mathcal{I}_{>N-k}$, define $f_{I}$ to be the greatest common divisor of the sublist $\alpha_{i}, i \in I$. Let $\mathcal{G}_{>N-k}(\boldsymbol{\alpha})=$ $\left\{f_{I}: I \in \mathcal{I}_{>N-k}\right\}$ be the set of integers so obtained. Because $\mathcal{I}_{>N-k}$ is stable by the operation of taking supersets, the set $\mathcal{G}_{>N-k}(\boldsymbol{\alpha})$ is a set of integers stable by the operation of taking greatest common divisors. Thus, $\mathcal{G}_{>N-k}(\boldsymbol{\alpha})$ can be considered as a poset (partially ordered set), where $f \preceq f^{\prime}$ if $f$ divides $f^{\prime}$.

Using the group $G(f) \subset \mathbb{C}^{\times}$of $f$-th roots of unity,

$$
G(f)=\left\{\zeta \in \mathbb{C}: \zeta^{f}=1\right\}
$$

we have thus $\mathcal{P}_{>N-k}=\bigcup_{f \in \mathcal{G}_{>N-k}(\boldsymbol{\alpha})} G(f)$; this is, of course, not a disjoint union. Then using the inclusion-exclusion principle, we can write the characteristic function of the set $\mathcal{P}_{>N-k}$ as a linear combination of characteristic functions of the sets $G(f)$ :

$$
\left[\mathcal{P}_{>N-k}\right]=\sum_{f \in \mathcal{G}_{>N-k}(\boldsymbol{\alpha})} \mu(f)[G(f)]
$$

where $\mu(f)$ are integers computed recursively. Such a function $\mu$ will be called as always a Möbius function for the poset (see Chapter 3 [15] for details on posets).

For fixed $k$, all the data above can be computed in polynomial time in function of the data $\alpha$. The greatest common divisor of a set of integers is computed in polynomial time. Finally the Möbius function 
$\mu(f)$ is computed in polynomial time, because there are polynomially many levels of the poset being considered.

Let us define for any positive integer $f$

$$
E(\boldsymbol{\alpha}, f)(t)=-\sum_{\zeta^{f}=1} \operatorname{Res}_{z=\zeta} z^{-t-1} F(\boldsymbol{\alpha})(z) \mathrm{d} z .
$$

Proposition 2.4. Let $k$ be a fixed integer, then

$$
E_{\mathcal{P}_{>N-k}}(t)=-\sum_{f \in \mathcal{G}_{>N-k}(\boldsymbol{\alpha})} \mu(f) E(\boldsymbol{\alpha}, f)(t)
$$

Thus we have reduced the computation to the fast computation of $E(\boldsymbol{\alpha}, f)(t)$. We will return to that in a moment but before we continue with the proof of Theorem 1.2, there are some interesting consequences for the classical theory of Denumerants.

Equation $(2.2)$ provides explicit expressions for the coefficients of the denumerant $E(\boldsymbol{\alpha})(t)$. In the past, researchers have discussed $E(\boldsymbol{\alpha})(t)$ in terms of its generating function (which belongs to the well-known clan of rational generating functions [15]), formulas for $E(\boldsymbol{\alpha})(t)$ in terms of binomial coefficients can be obtained using partial fraction decomposition. In [14] the authors propose another way to recover the coefficients of the quasipolynomial by a method they named rigorous guessing. In [14] quasipolynomials are represented as a function $f(t)$ given by $q$ polynomials $f^{[1]}(t), f^{[2]}(t), \ldots, f^{[q]}(t)$ such that $f(t)=$ $f^{[i]}(t)$ when $t \equiv i(\bmod q)$. To find the coefficients of the $f^{[i]}$ their method finds the first few terms of the Maclaurin expansion of the partial fraction decomposition to find enough evaluations of those polynomials and then recovers the coefficients of the $f^{[i]}$ as a result of solving a linear system. Our approach appeals instead to the number theoretic and polyhedral geometric nature of the problem and instead of $f^{[i]}$ 's we have a single expression whose coefficients are products of step polynomials.

\section{Polyhedral reinterpretation of the generating function $E(\boldsymbol{\alpha}, f)(t)$}

To complete the proof of Theorem 1.2 we need only to prove the following proposition.

Proposition 3.1. For any integer $f \in \mathcal{G}_{>N-k}(\boldsymbol{\alpha})$, the coefficient functions of the quasi-polynomial function $E(\boldsymbol{\alpha}, f)(t)$ and hence $E_{\mathcal{P}_{>N-k}}(t)$ are computed in polynomial time as step polynomials of $t$.

By the previous proposition we know we need to compute the value of $E(\boldsymbol{\alpha}, f)(t)$. Our goal now is to demonstrate that this function can be thought of as the generating function of the lattice points inside a convex cone. This is a key point to guarantee good computational bounds. Before we can do that we review some preliminaries on generating functions of cones. We recall the notion of generating functions of cones; see also [1].

Let $V=\mathbb{R}^{r}$ provided with a lattice $\Lambda$, and let $V^{*}$ denote the dual space. A (rational) simplicial cone $\mathfrak{c}=\mathbb{R}_{\geq 0} \mathbf{w}_{1}+\cdots+\mathbb{R}_{\geq 0} \mathbf{w}_{r}$ is a cone generated by $r$ linearly independent vectors $\mathbf{w}_{1}, \ldots, \mathbf{w}_{r}$ of $\Lambda$. We consider the semi-rational affine cone $\mathbf{s}+\mathfrak{c}, \mathbf{s} \in V$. Let $\boldsymbol{\xi} \in V^{*}$ be a dual vector such that $\left\langle\boldsymbol{\xi}, \mathbf{w}_{i}\right\rangle<0,1 \leq i \leq r$. Then the sum

$$
S(\mathbf{s}+\mathfrak{c}, \Lambda)(\boldsymbol{\xi})=\sum_{\mathbf{n} \in(\mathbf{s}+\mathfrak{c}) \cap \Lambda} \mathrm{e}^{\langle\boldsymbol{\xi}, \mathbf{n}\rangle}
$$


is summable and defines an analytic function of $\boldsymbol{\xi}$. It is well known that this function extends to a meromorphic function of $\boldsymbol{\xi} \in V_{\mathbb{C}}^{*}$. We still denote this meromorphic extension by $S(\mathbf{s}+\mathfrak{c}, \Lambda)(\boldsymbol{\xi})$.

Recall the following result.

Theorem 3.2. The series $S(\mathbf{s}+\mathfrak{c}, \Lambda)(\boldsymbol{\xi})$ is a meromorphic function of $\boldsymbol{\xi}$ such that $\prod_{i=1}^{r}\left\langle\boldsymbol{\xi}, \mathbf{w}_{i}\right\rangle S(\mathbf{s}+$ $\mathfrak{c}, \Lambda)(\boldsymbol{\xi})$ is holomorphic in a neighborhood of $\mathbf{0}$.

Let $\mathbf{t} \in \Lambda$. Consider the translated cone $\mathbf{t}+\mathbf{s}+\mathfrak{c}$ of $\mathbf{s}+\mathfrak{c}$ by $\mathbf{t}$. Then we have the covariance formula

$$
S(\mathbf{t}+\mathbf{s}+\mathfrak{c}, \Lambda)(\boldsymbol{\xi})=\mathrm{e}^{\langle\boldsymbol{\xi}, \mathbf{t}\rangle} S(\mathbf{s}+\mathfrak{c}, \Lambda)(\boldsymbol{\xi})
$$

Because of this formula, it is convenient to introduce the following function.

Definition 3.3. Define the function

$$
M(\mathbf{s}, \mathfrak{c}, \Lambda)(\boldsymbol{\xi})=\mathrm{e}^{-\langle\boldsymbol{\xi}, \mathbf{s}\rangle} S(\mathbf{s}+\mathfrak{c}, \Lambda)(\boldsymbol{\xi}) .
$$

Thus the function $\mathbf{s} \mapsto M(\mathbf{s}, \mathfrak{c}, \Lambda)(\boldsymbol{\xi})$ is a function of $\mathbf{s} \in V / \Lambda$ (a periodic function of $\mathbf{s}$ ) whose values are meromorphic functions of $\boldsymbol{\xi}$.

The function is easy to write down for a unimodular cone, that is a cone $\mathfrak{u}$ whose primitive generators $\mathbf{g}_{i}^{\mathfrak{u}}$ form a basis of the lattice $\Lambda$. We introduce the following notation.

Definition 3.4. Let $\mathfrak{u}$ be a unimodular cone with primitive generators $\mathbf{g}_{i}^{\mathfrak{u}}$ and let $\mathbf{s} \in V$. Then, write $\mathbf{s}=\sum_{i} s_{i} \mathbf{g}_{i}^{\mathfrak{u}}$, with $s_{i} \in \mathbb{R}$, and define

$$
\{-\mathbf{s}\}_{\mathfrak{u}}=\sum_{i}\left\{-s_{i}\right\} \mathbf{g}_{i}^{\mathfrak{u}}
$$

Thus $\mathbf{s}+\{-\mathbf{s}\}_{\mathfrak{u}}=\sum_{i}\left\lceil s_{i}\right\rceil \mathbf{g}_{i}^{\mathfrak{u}}$. Note that if $\mathbf{t} \in \Lambda$, then $\{-(\mathbf{s}+\mathbf{t})\}_{\mathfrak{u}}=\{-\mathbf{s}\}_{\mathfrak{u}}$. Thus, $\mathbf{s} \mapsto\{-\mathbf{s}\}_{\mathfrak{u}}$ is a function on $V / \Lambda$ with value in $V$. For any $\boldsymbol{\xi} \in V^{*}$, we then find

$$
S(\mathbf{s}+\mathfrak{u}, \Lambda)(\boldsymbol{\xi})=\mathrm{e}^{\langle\boldsymbol{\xi}, \mathbf{s}\rangle} \mathrm{e}^{\left\langle\boldsymbol{\xi},\{-\mathbf{s}\}_{\mathfrak{u}}\right\rangle} \frac{1}{\prod_{j}\left(1-\mathrm{e}^{\left\langle\boldsymbol{\xi}, \mathbf{g}_{j}^{u}\right\rangle}\right)}
$$

and thus

$$
M(\mathbf{s}, \mathfrak{u}, \Lambda)(\boldsymbol{\xi})=\mathrm{e}^{\left\langle\boldsymbol{\xi},\{-\mathbf{s}\}_{\mathfrak{u}}\right\rangle} \frac{1}{\prod_{j}\left(1-\mathrm{e}^{\left\langle\boldsymbol{\xi}, \mathbf{g}_{j}^{u}\right\rangle}\right)} .
$$

For a general cone $\mathfrak{c}$, we can decompose its characteristic function $[\mathfrak{c}]$ as a signed sum of characteristic functions of unimodular cones, $\sum_{\mathfrak{u}} \epsilon_{\mathfrak{u}}[\mathfrak{u}]$, modulo characteristic functions of cones containing lines. As shown by Barvinok, if the dimension $r$ of $V$ is fixed, this decomposition can be computed in polynomial time. Then we can write

$$
S(\mathbf{s}+\mathfrak{c}, \Lambda)(\boldsymbol{\xi})=\sum_{\mathfrak{u}} \epsilon_{\mathfrak{u}} S(\mathbf{s}+\mathfrak{u}, \Lambda)(\boldsymbol{\xi}) .
$$

Thus we obtain, using Formula 3.2],

$$
M(\mathbf{s}, \mathfrak{c}, \Lambda)(\boldsymbol{\xi})=\sum_{\mathfrak{u}} \epsilon_{\mathfrak{u}} \mathrm{e}^{\left\langle\boldsymbol{\xi},\{-\mathbf{s}\}_{\mathfrak{u}}\right\rangle} \frac{1}{\prod_{j}\left(1-\mathrm{e}^{\left\langle\boldsymbol{\xi}, \mathbf{g}_{i}^{\mathfrak{u}}\right\rangle}\right)} .
$$

Here $\mathfrak{u}$ runs through all the unimodular cones occurring in the decomposition of $\mathfrak{c}$, and the $\mathbf{g}_{i}^{\mathfrak{u}} \in \Lambda$ are the generators of the unimodular cone $\mathfrak{u}$. 
Remark 3.5. For computing explicit examples, it is convenient to make a change of variables that leads to computations in the standard lattice $\mathbb{Z}^{r}$. Let $B$ be the matrix whose columns are the generators of the lattice $\Lambda$; then $\Lambda=B \mathbb{Z}^{r}$.

$$
\begin{aligned}
M(\mathbf{s}, \mathfrak{c}, \Lambda)(\boldsymbol{\xi}) & =\mathrm{e}^{-\langle\boldsymbol{\xi}, \mathbf{s}\rangle} \sum_{\mathbf{n} \in(\mathbf{s}+\mathfrak{c}) \cap B \mathbb{Z}^{r}} \mathrm{e}^{\langle\boldsymbol{\xi}, \mathbf{n}\rangle} \\
& =\mathrm{e}^{-\left\langle B^{\top} \boldsymbol{\xi}, B^{-1} \mathbf{s}\right\rangle} \sum_{\mathbf{x} \in\left(B^{-1}(\mathbf{s}+\mathfrak{c}) \cap \mathbb{Z}^{r}\right.} \mathrm{e}^{\left\langle B^{\top} \boldsymbol{\xi}, \mathbf{x}\right\rangle}=M\left(B^{-1} \mathbf{s}, B^{-1} \mathfrak{c}, \mathbb{Z}^{r}\right)\left(B^{\top} \boldsymbol{\xi}\right) .
\end{aligned}
$$

\subsection{Back to the computation of $E(\boldsymbol{\alpha}, f)(t)$}

After the preliminaries we will see how to rewrite $E(\boldsymbol{\alpha}, f)(t)$ in terms of lattice points of cones. This will require some suitable manipulation of the initial form of $E(\boldsymbol{\alpha}, f)(t)$. So we introduce some notation. Let $k$ be fixed. For $f \in \mathcal{G}_{>N-k}(\boldsymbol{\alpha})$, define $\mathcal{F}(\boldsymbol{\alpha}, f, T)(x)=\sum_{\zeta^{f}=1} \frac{\zeta^{-T}}{\prod_{i=1}^{N+1}\left(1-\zeta^{\left.\alpha_{i} \mathrm{e}^{\alpha_{i} x}\right)}\right.}, \mathcal{E}(\boldsymbol{\alpha}, f)(t, T)=$ $-\operatorname{res}_{x=0} \mathrm{e}^{-t x} \mathcal{F}(\boldsymbol{\alpha}, f, T)(x)$, and $E_{i}(f)(T)=\operatorname{res}_{x=0} \frac{(-x)^{i}}{i !} \mathcal{F}(\boldsymbol{\alpha}, f, T)(x)$. Writing $z=\zeta \mathrm{e}^{x}$ and changing coordinates in residues, we obtain immediately:

$$
E(\boldsymbol{\alpha}, f)(t)=\left.\mathcal{E}(\boldsymbol{\alpha}, f)(t, T)\right|_{T=t}
$$

The dependence in $T$ of $\mathcal{F}(\boldsymbol{\alpha}, f, T)(x)$ is through $\zeta^{T}$. As $\zeta^{f}=1$, the function $\mathcal{F}(\boldsymbol{\alpha}, f, T)(x)$ is a periodic function of $T$ modulo $f$ whose values are meromorphic functions of $x$. Since the pole in $x$ is of order at most $N+1$, we can rewrite $\mathcal{E}(\boldsymbol{\alpha}, f)(t, T)$ in terms of $E_{i}(f)(T)$ and prove:

Theorem 3.6. Let $k$ be fixed. Then for $f \in \mathcal{G}_{>N-k}(\boldsymbol{\alpha})$ we can write

$$
\mathcal{E}(\boldsymbol{\alpha}, f)(t, T)=\sum_{i=0}^{N} t^{i} E_{i}(f)(T)
$$

with $E_{i}(f)(T)$ a step polynomial of degree less than or equal to $N-i$ and periodic of $T$ modulo $f$. This step polynomial can be computed in polynomial time.

For example $E_{N}$ is independent of $T$, thus it is a constant.

It is now clear that once we have proved Theorem 3.6, then the proof of Theorem 1.2 will follow. So we now concentrate on writing the function $\mathcal{F}(\boldsymbol{\alpha}, f, T)(x)$ more explicitly.

Definition 3.7. For a list $\alpha$ and integers $f$ and $T$, define meromorphic functions of $x \in \mathbb{C}$ by:

$$
\mathcal{B}(\boldsymbol{\alpha}, f)(x):=\frac{1}{\prod_{i: f \mid \alpha_{i}}\left(1-\mathrm{e}^{\alpha_{i} x}\right)}, \quad \mathcal{S}(\boldsymbol{\alpha}, f, T)(x):=\sum_{\zeta: \zeta^{f}=1} \frac{\zeta^{-T}}{\prod_{i: f \nmid \alpha_{i}}\left(1-\zeta^{\alpha_{i}} \mathrm{e}^{\alpha_{i} x}\right)} .
$$

Thus

$$
\mathcal{F}(\boldsymbol{\alpha}, f, T)(x)=\mathcal{B}(\boldsymbol{\alpha}, f)(x) \mathcal{S}(\boldsymbol{\alpha}, f, T)(x) .
$$

The expression we obtained will allow us to compute $\mathcal{F}(\boldsymbol{\alpha}, f, T)$ by relating $S(\boldsymbol{\alpha}, f, T)$ to a generating function of a cone. This cone will have fixed dimension when $k$ is fixed. 


\section{2 $E(\boldsymbol{\alpha}, f)(t)$ as the generating function of a cone in fixed dimension}

To this end, let $f$ be an integer from $\mathcal{G}_{>N-k}(\boldsymbol{\alpha})$. By definition, $f$ is the greatest common divisor of a sublist of $\boldsymbol{\alpha}$. Thus the greatest common divisor of $f$ and the elements of $\boldsymbol{\alpha}$ which are not a multiple of $f$ is still equal to 1 . Let $I=I(\boldsymbol{\alpha}, f)$ be the set of indices $i \in\{1, \ldots, N+1\}$ such that $\alpha_{i}$ is indivisible by $f$, i.e., $f \nmid \alpha_{i}$. Note that $f$ by definition is the greatest common divisor of all except at most $k$ of the integers $\alpha_{j}$. Let $r$ denote the cardinality of $I$; then $r \leq k$. Let $V_{I}=\mathbb{R}^{I}$ and let $V_{I}^{*}$ denote the dual space. We also define the sublist $\boldsymbol{\alpha}_{I}=\left[\alpha_{i}\right]_{i \in I}$ of elements of $\boldsymbol{\alpha}$ indivisible by $f$ and view it as a vector in $V_{I}^{*}$.

Definition 3.8. For an integer $T$, define the meromorphic function of $\boldsymbol{\xi} \in V_{I}^{*}$,

$$
Q(\boldsymbol{\alpha}, f, T)(\boldsymbol{\xi})=\sum_{\zeta: \zeta^{f}=1} \frac{\zeta^{-T}}{\prod_{j \in I(\boldsymbol{\alpha}, f)}\left(1-\zeta^{\alpha_{j}} \mathrm{e}^{\xi_{j}}\right)} .
$$

Remark 3.9. Observe that $Q(\boldsymbol{\alpha}, f, T)$ can be restricted at $\boldsymbol{\xi}=\boldsymbol{\alpha}_{I} x$, for $x \in \mathbb{C}$ generic, to give $\mathcal{S}(\boldsymbol{\alpha}, f, T)(x)$.

We find that $Q(\boldsymbol{\alpha}, f, T)(\boldsymbol{\xi})$ is the discrete generating function of an affine shift of the standard cone relative to a certain lattice in $V_{I}$, which we define as:

$$
\Lambda(\boldsymbol{\alpha}, f)=\left\{\mathbf{y} \in \mathbb{Z}^{I}:\left\langle\boldsymbol{\alpha}_{I}, \mathbf{y}\right\rangle=\sum_{j \in I} y_{j} \alpha_{j} \in \mathbb{Z} f\right\} .
$$

Consider the map $\phi: \mathbb{Z}^{I} \rightarrow \mathbb{Z} / \mathbb{Z} f, \mathbf{y} \mapsto\langle\boldsymbol{\alpha}, \mathbf{y}\rangle+\mathbb{Z} f$. Its kernel is the lattice $\Lambda(\boldsymbol{\alpha}, f)$. Because the greatest common divisor of $f$ and the elements of $\boldsymbol{\alpha}_{I}$ is 1, by Bezout's theorem there exist $s_{0} \in \mathbb{Z}$ and $\mathbf{s} \in \mathbb{Z}^{I}$ such that $1=\sum_{i \in I} s_{i} \alpha_{i}+s_{0} f$. Therefore, the map $\phi$ is surjective, and therefore the index $\left|\mathbb{Z}^{I}: \Lambda(\boldsymbol{\alpha}, f)\right|$ equals $f$.

Theorem 3.10. Let $\boldsymbol{\alpha}=\left[\alpha_{1}, \ldots, \alpha_{N+1}\right]$ be a list of positive integers and $f$ be the greatest common divisor of a sublist of $\boldsymbol{\alpha}$. Let $I=I(\boldsymbol{\alpha}, f)=\left\{i: f \nmid \alpha_{i}\right\}$. Let $s_{0} \in \mathbb{Z}$ and $\mathbf{s} \in \mathbb{Z}^{I}$ such that $1=$ $\sum_{i \in I} s_{i} \alpha_{i}+s_{0} f$ using Bezout's theorem. Let $T$ be an integer, and $\boldsymbol{\xi} \in V_{I}^{*}$. Then

$$
Q(\boldsymbol{\alpha}, f, T)(\boldsymbol{\xi})=f M\left(-T \mathbf{s}, \mathbb{R}_{\geq 0}^{I}, \Lambda(\boldsymbol{\alpha}, f)\right)(\boldsymbol{\xi}) .
$$

Remark 3.11. The function $Q(\boldsymbol{\alpha}, f, T)(\boldsymbol{\xi})$ is a function of $T$ periodic modulo $f$. Since $f \mathbb{Z}^{I}$ is contained in $\Lambda(\boldsymbol{\alpha}, f)$, the element $f \mathbf{s}$ is in the lattice $\Lambda(\boldsymbol{\alpha}, f)$, and we see that the right hand side is also a periodic function of $T$ modulo $f$.

of Theorem 3.10 Consider $\boldsymbol{\xi} \in V_{I}^{*}$ with $\xi_{j}<0$. Then we can write the equality

$$
\frac{1}{\prod_{j \in I}\left(1-\zeta^{\alpha_{j}} \mathrm{e}^{\xi_{j}}\right)}=\prod_{j \in I} \sum_{n_{j}=0}^{\infty} \zeta^{n_{j} \alpha_{j}} \mathrm{e}^{n_{j} \xi_{j}} . \quad \text { So } \quad Q(\boldsymbol{\alpha}, f, T)(\boldsymbol{\xi})=\sum_{\mathbf{n} \in \mathbb{Z}_{\geq 0}^{I}}\left(\sum_{f: \zeta^{f}=1} \zeta^{\sum_{j} n_{j} \alpha_{j}-T}\right) \mathrm{e}^{\sum_{j \in I} n_{j} \xi_{j}} .
$$

We note that $\sum_{f: \zeta^{f}=1} \zeta^{m}$ is zero except if $m \in \mathbb{Z} f$, when this sum is equal to $f$. Then we obtain that $Q(\boldsymbol{\alpha}, f, T)$ is the sum over $\mathbf{n} \in \mathbb{Z}_{\geq 0}^{I}$ such that $\sum_{j} n_{j} \alpha_{j}-T \in \mathbb{Z} f$. The equality $1=\sum_{j \in I} s_{j} \alpha_{j}+s_{0} f$ implies that $T \equiv \sum_{j} t s_{j} \alpha_{j}$ modulo $f$, and the condition $\sum_{j} n_{j} \alpha_{j}-T \in \mathbb{Z} f$ is equivalent to the condition $\sum_{j}\left(n_{j}-T s_{j}\right) \alpha_{j} \in \mathbb{Z} f$. 
We see that the point $\mathbf{n}-T \mathbf{s}$ is in the lattice $\Lambda(\boldsymbol{\alpha}, f)$ as well as in the cone $-T \mathbf{s}+\mathbb{R}_{\geq 0}^{I}$ (as $n_{j} \geq$ $0)$. Thus our function $Q(\boldsymbol{\alpha}, f, T)(\boldsymbol{\xi})$ is equal to $f \mathrm{e}^{\langle\boldsymbol{\xi}, T \mathbf{s}\rangle} S\left(-T \mathbf{s}+\mathbb{R}_{\geq 0}^{I}, \Lambda(\boldsymbol{\alpha}, f)\right)(\boldsymbol{\xi})=\bar{f} M(-T \mathbf{s}+$ $\left.\mathbb{R}_{\geq 0}^{I}, \Lambda(\boldsymbol{\alpha}, f)\right)(\boldsymbol{\xi})$.

\subsection{Unimodular decomposition in the dual space}

The cone $\mathbb{R}_{\geq 0}^{I}$ is in general not unimodular with respect to the lattice $\Lambda(\boldsymbol{\alpha}, f)$. By decomposing $\mathbb{R}_{\geq 0}^{I}$ in cones $\mathfrak{u}$ that are unimodular with respect to $\Lambda(\boldsymbol{\alpha}, f)$, modulo cones containing lines, we can write $M\left(-T \mathbf{s}, \mathbb{R}_{>0}^{I}, \Lambda(\boldsymbol{\alpha}, f)\right)=\sum_{\mathfrak{u}} \epsilon_{\mathfrak{u}} M(-T \mathbf{s}, \mathfrak{u}, \Lambda)$, where $\epsilon_{\mathfrak{u}} \in\{ \pm 1\}$. This decomposition can be computed using Barvinok's algorithm in polynomial time for fixed $k$ because the dimension $|I|$ is at most $k$.

Remark 3.12. Although we know that the meromorphic function $M\left(-T \mathbf{s}, \mathbb{R}_{\geq 0}^{I}, \Lambda(\boldsymbol{\alpha}, f)\right)(\boldsymbol{\xi})$ restricts via $\boldsymbol{\xi}=\boldsymbol{\alpha}_{I} x$ to a meromorphic function of a single variable $x$, it may happen that the individual functions $M(-T \mathbf{s}, \mathfrak{u}, \Lambda(\boldsymbol{\alpha}, f))(\boldsymbol{\xi})$ do not restrict. In other words, the line $\boldsymbol{\alpha}_{I} x$ may be entirely contained in the set of poles. If this is the case, we can compute (in polynomial time) a regular vector $\boldsymbol{\beta} \in \mathbb{Q}^{I}$ so that all functions $M(-T \mathbf{s}+\mathfrak{u}, \Lambda(\boldsymbol{\alpha}, f))(\boldsymbol{\xi})$ occurring can be evaluated on $\left(\boldsymbol{\alpha}_{I}+\epsilon \boldsymbol{\beta}\right) x$.

Finally let us analyze the dependence in $T$ of the functions $M(-T \mathbf{s}, \mathfrak{u}, \Lambda(\boldsymbol{\alpha}, f))$, where $\mathfrak{u}$ is a unimodular cone. Let the generators be $\mathbf{g}_{i}^{\mathfrak{u}}$, so the elements $\mathbf{g}_{i}^{\mathfrak{u}}$ form a basis of the lattice $\Lambda(\boldsymbol{\alpha}, f)$. Recall that the lattice $f \mathbb{Z}^{r}$ is contained in $\Lambda(\boldsymbol{\alpha}, f)$. Thus as $\mathbf{s} \in \mathbb{Z}^{r}$, we have $\mathbf{s}=\sum_{i} s_{i} \mathbf{g}_{i}^{\mathrm{u}}$ with $f s_{i} \in \mathbb{Z}$ and hence $\{-T \mathbf{s}\}_{\mathfrak{u}}=\sum_{i}\left\{-T s_{i}\right\} \mathbf{g}_{i}^{\mathfrak{u}}$ with $\left\{-T s_{i}\right\}$ a function of $T$ periodic modulo $f$.

Thus the function $T \mapsto\{-T \mathbf{s}\}_{\mathfrak{u}}$ is a step linear function, modulo $f$, with value in $V$. We then write $M(-T \mathbf{s}, \mathfrak{u})(\boldsymbol{\xi})=\mathrm{e}^{\left\langle\boldsymbol{\xi},\{T \mathbf{s}\}_{\mathfrak{u}}\right\rangle} \prod_{j=1}^{r} \frac{1}{\left(1-\mathrm{e}^{\left\langle\boldsymbol{\xi}, \mathbf{g}_{j}\right\rangle}\right)}$, and hence finally

$$
\mathcal{F}(\boldsymbol{\alpha}, f, T)(x)=f M\left(-T \mathbf{s}, \mathbb{R}_{\geq 0}^{I}, \Lambda(\boldsymbol{\alpha}, f)\right)\left(\boldsymbol{\alpha}_{I} x\right) \prod_{j: f \mid \alpha_{j}} \frac{1}{\left(1-\mathrm{e}^{\alpha_{j} x}\right)} .
$$

This is a meromorphic function of the variable $x$. Near $x=0$, it is of the form $\sum_{\mathfrak{u}} \exp \left\{l_{\mathfrak{u}}(T) x\right\} h(x) / x^{N+1}$ where $h(x)$ is holomorphic in $x$ and $l_{\mathfrak{u}}(T)$ is a step linear function of $T$, modulo $f$. Thus to compute

$$
E_{i}(f)(T)=\operatorname{res}_{x=0} \frac{(-x)^{i}}{i !} \mathcal{F}(\boldsymbol{\alpha}, f, T)(x)
$$

we only have to expand the function $x \mapsto \exp \left\{l_{\mathfrak{u}}(T) x\right\}$ up to the power $x^{N-i}$. This expansion can be done in polynomial time. We thus see that as stated in Theorem 3.6, $E_{i}(f)(T)$ is a step polynomial of degree less than or equal to $(N-i)$, which is periodic of $T$ modulo $f$. This completes the proof of Theorem 3.6 and thus the proof of Theorem 1.2

\section{References}

[1] V. Baldoni, N. Berline, J. A. De Loera, M. Köppe, and M. Vergne, Computation of the highest coefficients of weighted Ehrhart quasi-polynomials of rational polyhedra, Foundations of Computational Mathematics (2011), Published online 12 November 2011.

[2] A. I. Barvinok, Polynomial time algorithm for counting integral points in polyhedra when the dimension is fixed, Mathematics of Operations Research 19 (1994), 769-779. 
[3] _ Computing the Ehrhart quasi-polynomial of a rational simplex, Math. Comp. 75 (2006), no. $255,1449-1466$.

[4] __ Integer points in polyhedra, Zürich Lectures in Advanced Mathematics, European Mathematical Society (EMS), Zürich, Switzerland, 2008.

[5] M. Beck, I. M. Gessel, and T. Komatsu, The polynomial part of a restricted partition function related to the Frobenius problem, Electron. J. Combin. 8 (2001), no. 1, Note 7, 5 pp. (electronic). MR 1855876 (2002f:05017)

[6] E. T. Bell, Interpolated denumerants and Lambert series, Amer. J. Math. 65 (1943), 382-386. MR $0009043(5,92 a)$

[7] L. Comtet, Advanced combinatorics, enlarged ed., D. Reidel Publishing Co., Dordrecht, 1974, The art of finite and infinite expansions. MR 0460128 (57 \#124)

[8] E. Ehrhart, Polynômes arithmétiques et méthode des polyèdres en combinatoire, Birkhäuser Verlag, Basel, 1977, International Series of Numerical Mathematics, Vol. 35. MR 0432556 (55 \#5544)

[9] D. Einstein, D. Lichtblau, A. Strzebonski, and S. Wagon, Frobenius numbers by lattice point enumeration, Integers 7 (2007), A15, 63. MR 2299816 (2008a:11031)

[10] R. Kannan, Lattice translates of a polytope and the Frobenius problem, Combinatorica 12 (1992), no. 2, 161-177. MR 1179254 (93k:52015)

[11] P. Lisoněk, Denumerants and their approximations, J. Combin. Math. Combin. Comput. 18 (1995), 225-232. MR 1334651 (96a:05009)

[12] J. L. Ramírez Alfonsín, The Diophantine Frobenius problem, Oxford Lecture Series in Mathematics and its Applications, vol. 30, Oxford University Press, Oxford, 2005. MR 2260521 (2007i:11052)

[13] J. Riordan, An introduction to combinatorial analysis, Dover Publications Inc., Mineola, NY, 2002, Reprint of the 1958 original [Wiley, New York; MR0096594 (20 \#3077)]. MR 1949650

[14] A. Sills and D. Zeilberger, Formula for the number of partitions of $n$ into at most $m$ parts (using the quasi-polynomial ansatz), Adv. in Appl. Math. 48 (2012), 640-645.

[15] R. P. Stanley, Enumerative combinatorics, vol. I, Cambridge, 1997. 